\title{
8 Effekte der Universitäten auf den Konferenztourismus der Stadt Wien
}

Der nationale und internationale Kongresstourismus stellt einen wichtigen Wirtschaftsfaktor für die Stadtökonomie Wiens dar. Gemäß der Statistik von Vienna Convention Bureau fanden 2011 in Wien 3.151 wissenschaftliche und nichtwissenschaftliche Kongresse statt, an denen rund 475.300 Teilnehmer aus dem In- und Ausland teilgenommen haben (VCB 2012, 10). Etwas weniger als ein Drittel davon (1.018 Veranstaltungen, 32,3\%) waren wissenschaftliche Veranstaltungen, die jedoch mit 428.787 Teilnehmern und rund 1,1 Mio. Nächtigungen einen überproportional hohen Beitrag zur Wiener Tourismuswirtschaft leisteten - immerhin ein Zehntel (9,8 \%) der 11,4 Mio. Nächtigungen durch Touristen im Jahr 2011. Der wissenschaftliche Kongresstourismus brachte der Stadt Wien einen Beitrag zum regionalen BIP von 673,70 Mio. Euro (VCB 2012, 26). Die Bedeutung der Kongresswirtschaft für Wien wird auch dadurch unterstrichen, dass die Stadt in der internationalen ICCA-Statistik 2010 wiederholt weltweit auf Platz 1 gerankt wird ${ }^{1}$.

Welchen Beitrag leisten die Wiener Hochschulen als Organisatoren und Veranstalter wissenschaftlicher Tagungen zu diesem für die Stadt Wien so bedeutenden Kongresstourismus? Hier ist zu bedenken, dass die wissenschaftlichen Veranstaltungen an den Universitäten hinsichtlich ihrer internationalen Orientierung, ihrer Größe und Dauer sehr heterogen sind. So zählte beispielsweise an der Universität Wien die größte Veranstaltung im Jahr 2011 rund 800 Teilnehmer („European Conference on Complex Systems“, ECCS11) und erstreckte sich über sechs Tage, während der 1-tägige Workshop „Vier Jahrzehnte China-Stipendien“ als kleinste Veranstaltung nur 15 Teilnehmer zählte. An der Universität für Bodenkultur war 2011 das „ESACT Meeting“ mit rund 1.000 Teilnehmern die größte Konferenz, das „BOKU Führungskräfteseminar“ und der „WISER-Workshop“ waren mit je 15 Teilnehmern die kleinsten Veranstaltungen. Eine Schwierigkeit für die Bewertung der Bedeutung der Wiener Hochschulen für den Konferenztourismus lag in der beschränkten Datenverfügbarkeit: nur zwei Universitäten (Universität Wien, Universität für Bodenkultur) machten hierzu Angaben.

Um die Bedeutung zu schätzen, wurde das Verhältnis für die Veranstaltungen, die Teilnehmer sowie die Summe der Veranstaltungstage dieser zwei Universitäten zu den dort tätigen Professoren hochgerechnet; diese Verhältniszahl wurde auf die restlichen Wiener Universitäten, denen eine gleiche Professoren-Veranstaltungsdichte unterstellt wurde, hochgerechnet. Da kaum Informationen über Fachhochschulen und Privatuniversitäten vorlagen, wurden diese von der Schätzung ausgenommen. Das Ergebnis der Schätzung für die Wiener Universitäten ist in Tabelle 8.1 dargestellt, wobei hier sämtliche gemeldete Veranstaltungen - vom inter-

vgl. www.wieninternational.at/de/content/wien-weltweit-kongressstadt-nummer-1-de (Zugriff 15. August 2013) 
nationalen Kongress bis zum Workshop - berücksichtigt worden sind. Es erfolgte lediglich eine Differenzierung nach nationalen und internationalen Veranstaltungen, wobei die Kongresssprache für die Zuordnung (deutsch/nicht-deutsch) ausschlaggebend war.

Tabelle 8.1: Veranstaltungen an den Universitäten Wiens 2011

\begin{tabular}{lrrr}
\hline & \multicolumn{3}{c}{ Universitäten Gesamt } \\
\cline { 2 - 4 } & gesamt & national & international \\
\hline Veranstaltungen & 868 & 395 & 473 \\
Teilnehmer & 103.738 & 42.843 & 60.895 \\
$\varnothing$ Teilnehmer je Veranstaltung & 119,5 & 108,6 & 128,6 \\
$\varnothing$ Dauer der Veranstaltungen & 2,3 & 1,9 & 2,6 \\
Summe Veranstaltungstage & 2.043 & 811 & 1.232 \\
Teilnehmertage (ø Teilnehmer x Tage) & 240.365 & 83.365 & 156.209 \\
\hline
\end{tabular}

Quelle: eigene Erhebung 2013; eigene Berechnungen.

Gemäß dieser Schätzung fanden an den Wiener Universitäten 2011 insgesamt 868 Veranstaltungen mit 103.738 Teilnehmern statt, wobei an jeder Veranstaltung im Schnitt 119,5 Wissenschaftler teilnahmen und diese im Schnitt 2,3 Tage dauerten (vgl. Tabelle 8.1). Internationale Veranstaltungenüberwiegenhinsichtlich derAnzahl(473 oder $54,5 \%$ ) sowie der Teilnehmerzahl (60.895 oder 58,7 \%), sie sind im Durchschnitt auch größer und haben eine längere Dauer als nationale Veranstaltungen. Rechnet man die Summe der Veranstaltungstage mit der durchschnittlichen Teilnehmeranzahl hoch, so ergeben sich für die Wiener Universitäten 240.365 Teilnehmertage, wobei die internationalen Veranstaltungen knapp zwei Drittel (64,9 \%) ausmachen. Die Bedeutung lässt sich nicht zuletzt daran ermessen, dass das VCB für das Jahr 2011 in Wien in Summe 903.634 Teilnehmertage bei nationalen und internationalen Kongressen gezählt hat. Wenn auch ein direkter Vergleich nicht möglich ist, dass die Kongresse gemäß VCB genau definiert sind und die zahlreichen kleineren und mittleren Veranstaltungen (Workshops, Seminare, Kolloquien u.Ä.) der Universitäten nicht berücksichtigt werden können, so liegt das Verhältnis VCB-Kongresse zu allen Veranstaltungen der Universitäten immerhin bei $1: 3,76$.

Um die unmittelbaren Wertschöpfungseffekte der wissenschaftlichen Veranstaltungen der Wiener Universitäten abschätzen zu können, kann auf die Erfahrungswerte der Wiener Kongressstatistik des Vienna Congress Bureau zurückgegriffen werden. Diese hat ergeben, dass der Wertschöpfungseffekt pro Teilnehmertag für nationale Kongresse bei 306 Euro, bei internationalen Kongressen bei 886 Euro liegt (VCB 2012). Die Übertragung auf die Veranstaltungen der Wiener Universitäten ist insofern schwierig, als der Veranstaltungstyp „Kongress“ an den Universitäten nicht wie bei der VCB definiert ist. Ebenso werden nationale und internationale Veranstaltungen an den Universitäten an- 
ders abgegrenzt. Weiters werden von der VCB keine Angaben zum Ausgabeverhalten der Kongressteilnehmer gemacht - was eine näherungsweise Adaptierung der Wertschöpfungskennzahlen erleichtern würde - sondern nur die mittels eines ökonometrischen Modells hochgerechneten Wertschöpfungsdaten publiziert (VCB 2012, 26).

Um dennoch eine vorsichtige Schätzung des Wertschöpfungseffektes der wissenschaftlichen Veranstaltungen durchführen zu können, werden als Grundlage nur Konferenzen und Kongresse herangezogen, also 204 der 868 Veranstaltungen. Dabei handelt es sich um 23,5\% der wissenschaftlichen Veranstaltungen, auf die jedoch 40,4\% der Teilnehmer entfallen; aufgrund der überdurchschnittlichen Größe und Dauer der Veranstaltungen (vgl. Tabelle 8.2) handelt es sich um knapp die Hälfte der Teilnehmertage (48,2 \% oder 115.796). Auf diese Teilnehmertage wird jener Wertschöpfungseffekt hochgerechnet, den das VCB für die nationalen Kongresse ermittelt hat. Diese konservative Annahme kann dadurch gerechtfertigt werden, dass die Abgrenzung zwischen nationalen und internationalen Kongressen an den Universitäten fließend ist und zweitens weniger kommerziell als jene Veranstaltungen, die vom VCB als Konferenz definiert werden. Damit ergibt sich für die Wiener Universitäten ein Wertschöpfungseffekt von etwa 36,7 Mio. Euro, davon 31,1 Mio. Euro für internationale Konferenzen und Kongresse. Gemessen an den 673,6 Mio. Euro, die die wissenschaftlichen Kongresse der Stadt Wien jährlich an Wertschöpfung bringen (VCB 2012, 26), erscheinen die durch die Universitäten induzierten Effekte möglicherweise gering. Dem ist jedoch entgegenzuhalten, dass es sich hier um endogene, von einem regionalen Akteur geschaffene Wertschöpfung handelt, die daher auch nicht Gefahr läuft, im nächsten Jahr vielleicht an einen anderen Standort abzuwandern.

Tabelle 8.2: Kongresse und Konferenzen an den Universitäten Wiens 2011, Schätzung der Wertschöpfung nach VCB-Berechnung (national)

\begin{tabular}{lrrr}
\hline & \multicolumn{3}{c}{ Universitäten Gesamt } \\
\cline { 2 - 4 } & \multicolumn{1}{c}{ gesamt } & national & international \\
\hline Veranstaltungen & 204 & 78 & 126 \\
Teilnehmer & 41.962 & 10.392 & 31.569 \\
$\varnothing$ Teilnehmer je Veranstaltung & 205,5 & 133,4 & 250,0 \\
$\varnothing$ Dauer der Veranstaltungen & 2,8 & 1,8 & 3,2 \\
Summe Veranstaltungstage & 547 & 159 & 389 \\
Teilnehmertage (ø Teilnehmer x Tage) & 115.796 & 18.343 & 101.524 \\
BIP Wertschöpfung nach VCB & 36.679 .273 & 5.612 .805 & 31.066 .468 \\
\hline
\end{tabular}

Quellen: eigene Erhebung 2013; eigene Berechnungen. 